\title{
Research Article New Classes of Graceful Trees
}

\author{
Md. Forhad Hossain, Md. Momin Al Aziz, and M. Kaykobad \\ Department of Computer Science and Engineering, Bangladesh University of Engineering and Technology, Dhaka 1000, Bangladesh
}

Correspondence should be addressed to Md. Momin Al Aziz; momin.aziz.cse@gmail.com

Received 18 July 2014; Revised 29 October 2014; Accepted 2 November 2014; Published 23 November 2014

Academic Editor: Aleksandar Ilić

Copyright (C) $2014 \mathrm{Md}$. Forhad Hossain et al. This is an open access article distributed under the Creative Commons Attribution License, which permits unrestricted use, distribution, and reproduction in any medium, provided the original work is properly cited.

Graceful labeling is one of the most researched problems. One of the earliest results is that caterpillars are graceful. We show that caterpillars connected to a vertex recursively satisfying certain conditions are also graceful.

\section{Introduction}

Let $T$ be any arbitrary tree on $m$ edges. If its vertices can be distinctly labeled using integers $0,1, \ldots, m$ so that all the induced edge labels (vertices labeled $i$ and $j$ induce label $|i-j|$ on edge $(i, j))$ are also distinct, then the labeling is called graceful. It was conjectured that all trees are graceful. This has connection with Ringel's conjecture and some related results can be found in [1, 2]; Kotzig later conjectured that such a decomposition could be achieved cyclically. Rosa [2] established that these conjectures could be solved by showing that every tree is graceful.

The conjecture has been very widely studied and new classes of trees have been proved to be graceful (see [3-5]). However, it has not been possible to devise a single algorithm to stand out against the conjecture for any arbitrary tree. The research has continued in two directions: newer and more generalized classes of graceful trees are discovered [6-8] or graceful labeling has been computed for all larger trees up to a fixed order. Computationally, it has been shown so far that all trees with up to 35 vertices are graceful [9]. Graceful labeling has found useful applications in coding theory, Xray crystallography, radar, astronomy, circuit design, communication network addressing, and database management (see $[10,11])$

In Section 2, we define the classes of trees that have already been proved graceful and critical to our work. Section 3 defines our work of two new classes of trees that have been proved graceful.

\section{Gracefully Labeled Trees}

Let us introduce the classes of trees for which graceful labeling has been found.

A path is a tree with only two leaves. A caterpillar is a tree such that if all leaves are removed, the remaining graph is a path. This path can be termed as backbone of the caterpillar. Rosa [12] proved that all caterpillars are graceful. In Figure 1, a gracefully labeled caterpillar has been shown. A caterpillar is labeled from one end of its backbone with 0 . Its adjacent vertices are labeled using so far unused largest labels in such a way that vertices on the path get alternately the largest and smallest labels. In this way, while we label vertices the largest unused edge labels are generated. It may be noted that for an $m$-edge tree if $f(i)$ is a graceful labeling, then so is $m-f(i)$. So we can label any end of a caterpillar by 0 or by $m$.

A rooted tree in which every level contains vertices of the same degree is called symmetrical trees. Bermond and Schönheim [13] proved that all symmetrical trees are graceful as in Figure 2.

In this paper, we prove that super-caterpillars obtained by joining a set of caterpillars of a special type to a vertex are also graceful (Figures 4 and 5).

\section{New Classes of Graceful Trees}

Let us introduce the following classes of trees.

Definition 1. Let $T_{0}$ be any arbitrary caterpillar and let $T_{i}$, $i=1, \ldots, k$, be caterpillars with $\left|T_{i}\right|=m$ number of vertices 


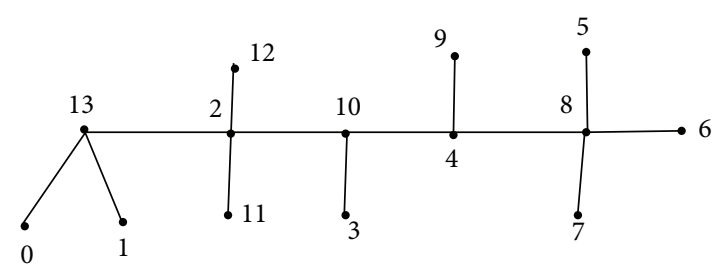

FIGURE 1: An example of gracefully labeled 14-vertex caterpillar.

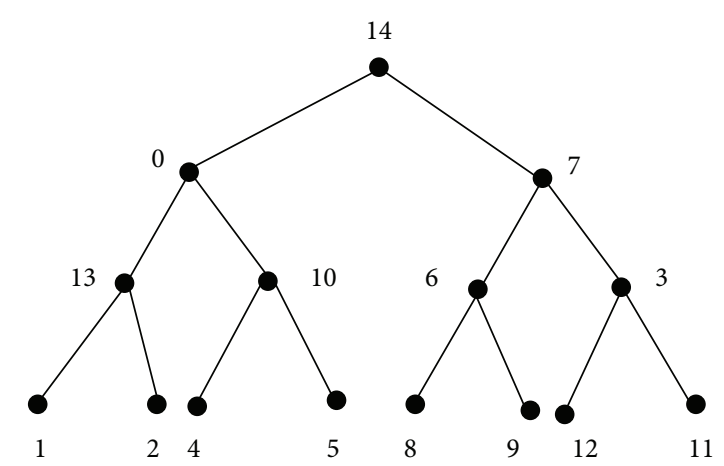

Figure 2: An example of gracefully labeled 15-vertex symmetrical tree.

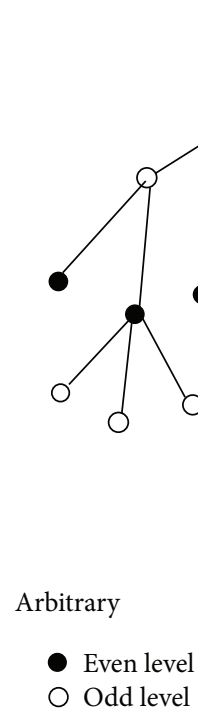

Even level
Odd level

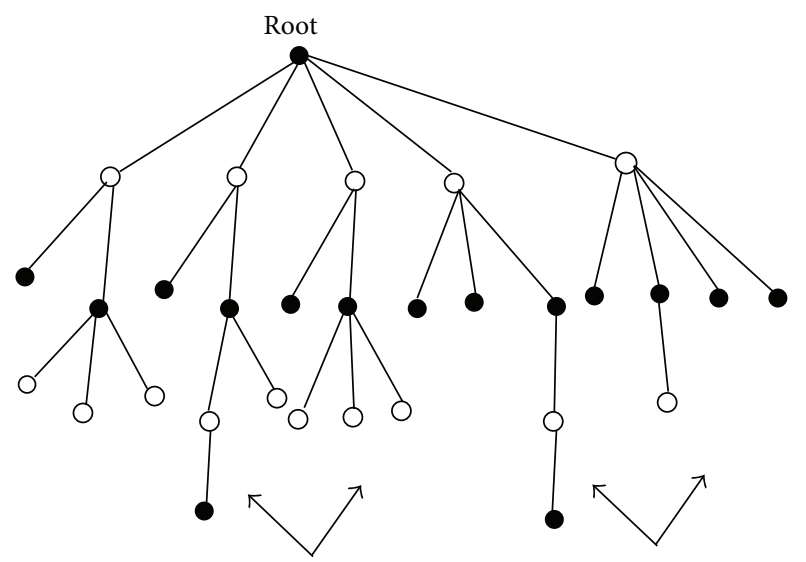

Pair
Pair
- Even level

O Odd level

(a)

(b)

FIGURE 3: Example of a super-caterpillar with (a) odd $k$ and (b) even $k$, with an arbitrary caterpillar joined with a root.

and the sum of vertex numbers is the same in odd levels of all pairs $T_{2 i+1}$ and $T_{2 i+2}$. In case of $k$ being an odd number, one caterpillar will be without a pair. Let a leaf attached to backbone of each $T_{i}$ be joined to a vertex $v$ by an edge. Then the resulting tree is called a super-caterpillar.

Two super-caterpillars are illustrated in Figure 3 with odd and even $k$. Now we have the following result.

Theorem 2. All super-caterpillars are graceful.
Proof of Theorem 2. Let us assume for simplicity that we have $k$ caterpillars joined to $v$ and that each caterpillar has the same number $m$ of vertices, also a total of $s_{i}$ vertices in odd levels, $s_{i}$ being equal for each pair of caterpillars $T_{2 i+1}$ and $T_{2 i+2}$. We also denote the vertex of $T_{j}$ connected to $v$ by $v_{j}$. Let us label $v$ by 0 . Now vertices of caterpillars will be in turn labeled using the smallest and largest labels following caterpillar-labeling schemes so that edge numbers are generated in descending order. The endpoint of $T_{1}$ connected to $v$ is labeled $\mathrm{km}$, and we will use up $m$ labels in $T_{1}$ of which $s_{1}$ large, namely, $\mathrm{km}, \mathrm{km}-1$, 


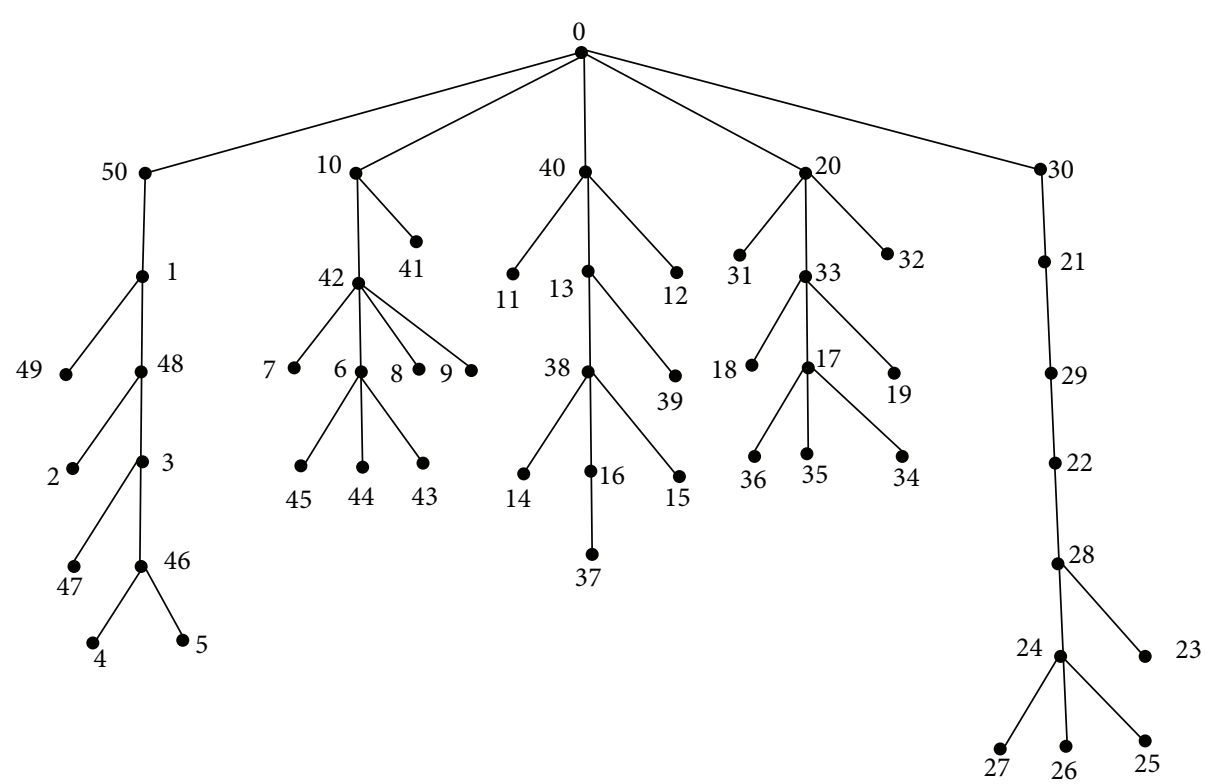

FIGURE 4: Example of a super-caterpillar gracefully labeled.

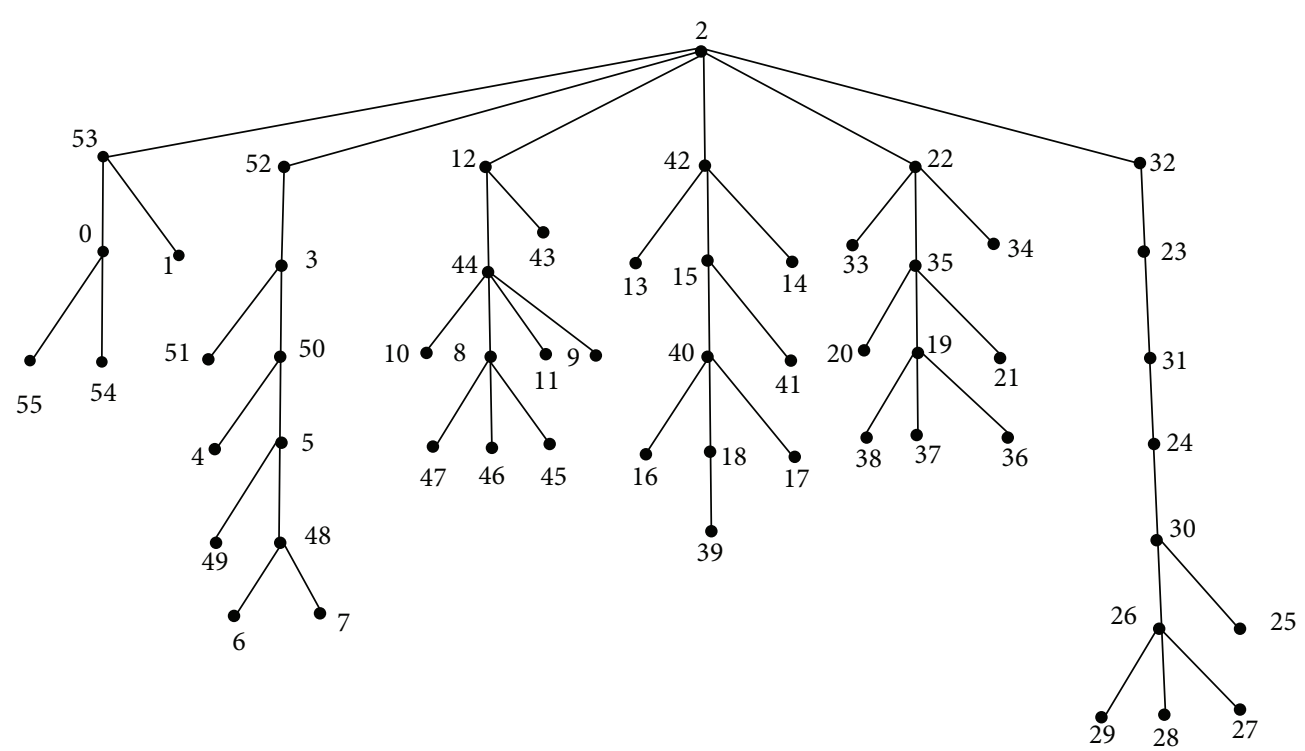

FIGURE 5: Example of super-caterpillar gracefully labeled with an arbitrary caterpillar where $s_{0}=2$.

$\ldots, k m-s_{1}+1$, and $m-s_{1}$ small labels, namely, $1, \ldots, m-s_{1}$. Thus the last edge label created is $k m-s_{1}+1-\left(m-s_{1}\right)=(k-$ $1) m+1$. In the next caterpillar, both the smallest and largest labels will differ by 1 from the labels used in the previous tree. In particular, one vertex will get label $\mathrm{km}-s_{1}-1$ and the other one $m-s_{1}$ resulting in edge label $k m-s_{1}-\left(m-s_{1}+1\right)=$ $(k-1) m-1$ missing the label $(k-1) m$. We are going to use up $m-s_{2}$ large and $s_{2}$ small labels in $T_{2}$ in bottom up fashion. Note that $s_{2}=s_{1} . T_{2}$ will be labeled in such a way that $v_{2}$ ends getting the smallest unused label, that is, label $m$. In this way in tandem vertices of odd levels will be numbered by big and small numbers, respectively, for odd and even indexed caterpillars. This will result in label $\left(v_{i}\right)=(k-\lfloor(i-1) / 2\rfloor) m$ for odd $i$, whereas label $\left(v_{i}\right)=i m / 2$ is for even ones. For each pair of caterpillars, labels $(k-\lfloor(i-1) / 2\rfloor) m$ and $i m / 2$ will be missing which will be generated on edges incident to vertex $v$.

Now assume that we have one arbitrary caterpillar $T_{0}$ and any $k$ caterpillars having the same number of vertices $m$ and that the last caterpillar has $m$ vertices but not necessarily having equal number of vertices in odd levels as all previous pairs have. One end of backbone of each caterpillar is connected to $v$. So total number of edges in the tree will be $\mathrm{km}+m_{0}$. Now start labeling $T_{0}$ in such a way that we end up labeling $v$ by $s_{0}$ shifting label of $v$ by $s_{0}$. Large labels have also been shifted by $m_{0}-\left(m_{0}-s_{0}\right)=s_{0}$, thus producing 


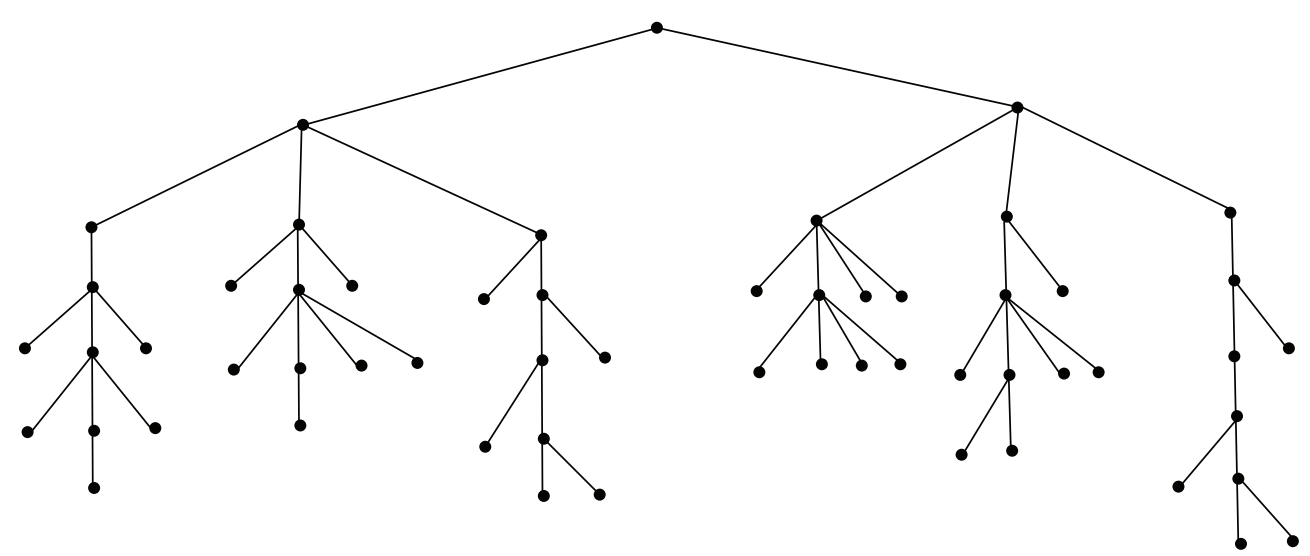

FIGURE 6: Example of an extended super-caterpillar with two groups $(k=2)$ consisting of three caterpillars.
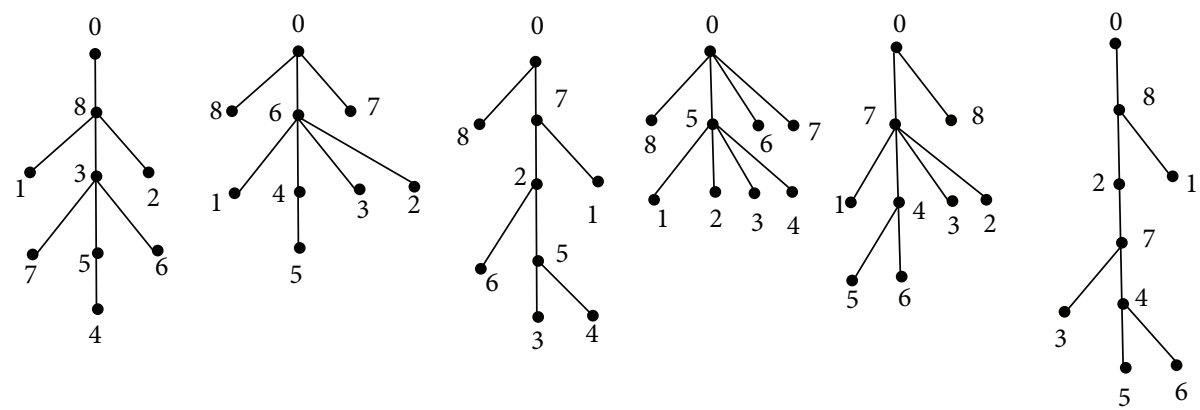

FIGURE 7: Six graceful caterpillars.
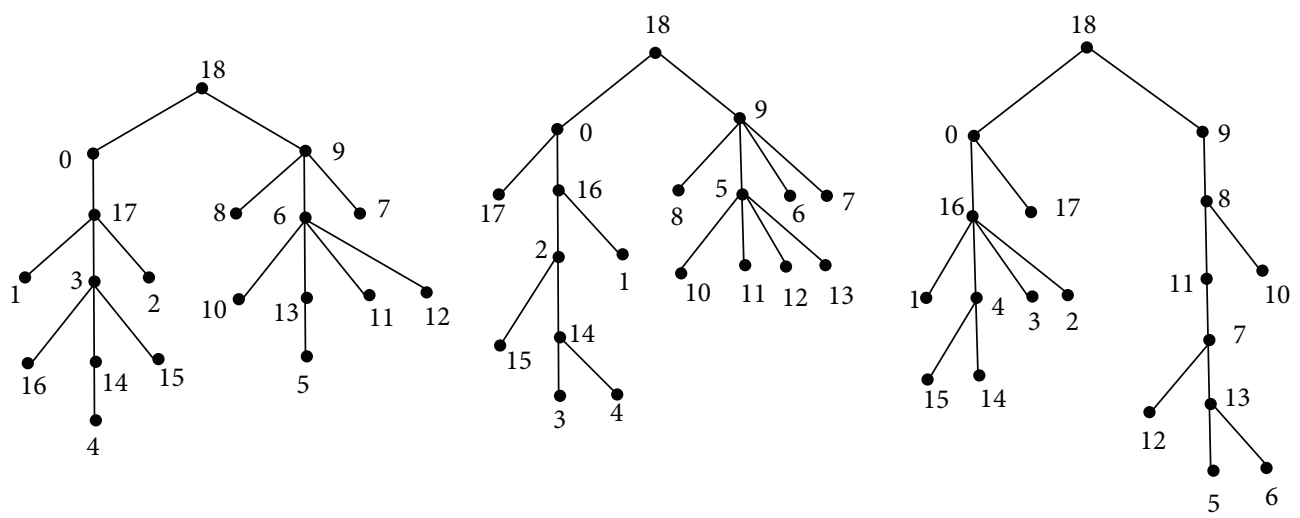

Figure 8: Graceful labeling of three grouped caterpillars which are joined to a single vertex $v_{i}$.

$(k-\lfloor(i-1) / 2\rfloor) m$ labels on the edge incident to $v$, which we have missed in moving from one caterpillar to the next. Now for $T_{1}$ we are left with numbers from $s_{0}+1$ to $k m+m_{0}-m+s_{0}=$ $(k-1) m+m_{0}+s$. This way all $v_{i}$ s, adjacent to root $v$, will be labeled by multiples of $m$. After we have completed labeling all pairs of caterpillars, we will be left with consecutive integers to label the vertices of the unpaired caterpillar and generate the remaining smallest possible edge labels.

Definition 3. Let there be any numbers of $k p$ caterpillars each having $m$ vertices and total number of vertices in odd (or even) levels of these caterpillars are the same. These caterpillars are grouped in $k$ groups each having $p$ caterpillars. Let backbones of each group $i$ of caterpillars be connected to vertex $v_{i}$ that is connected to vertex $v$. Then the resulting tree is called an extended super-caterpillar.

An extended super-caterpillar is illustrated in Figure 6 with six caterpillars grouped in two $(k=2)$, where each group has three caterpillars $(p=3)$ joined with a vertex $v_{i}$. Each caterpillar contains nine vertices $(s=9)$. Now we have the following result. 


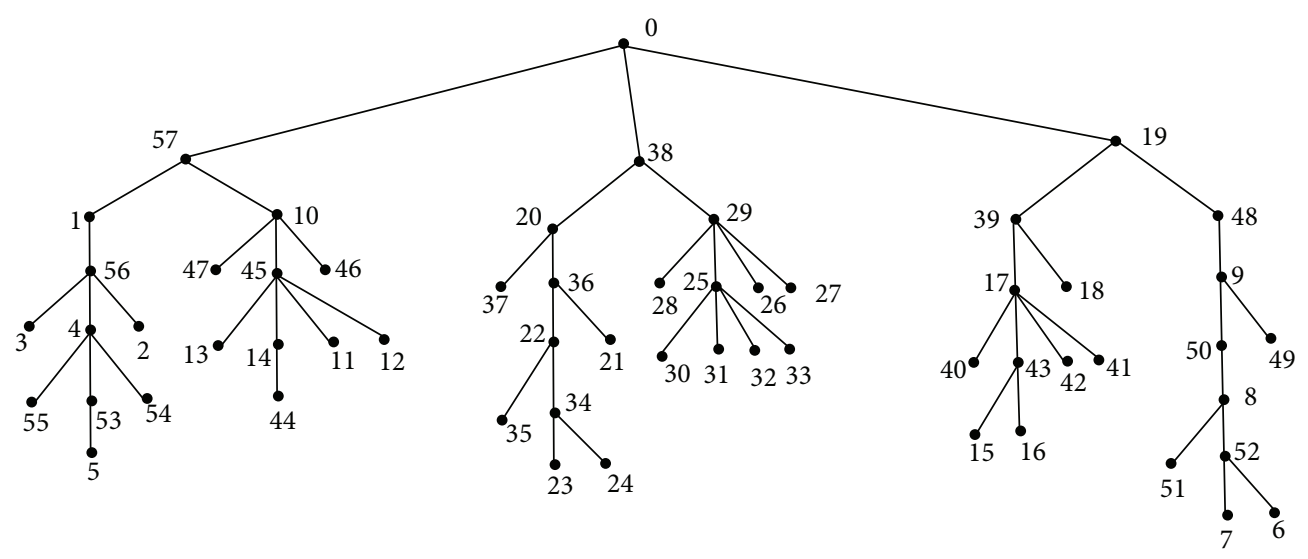

FIgURE 9: Graceful labeling of an extended super-caterpillar with root at vertex $v$.

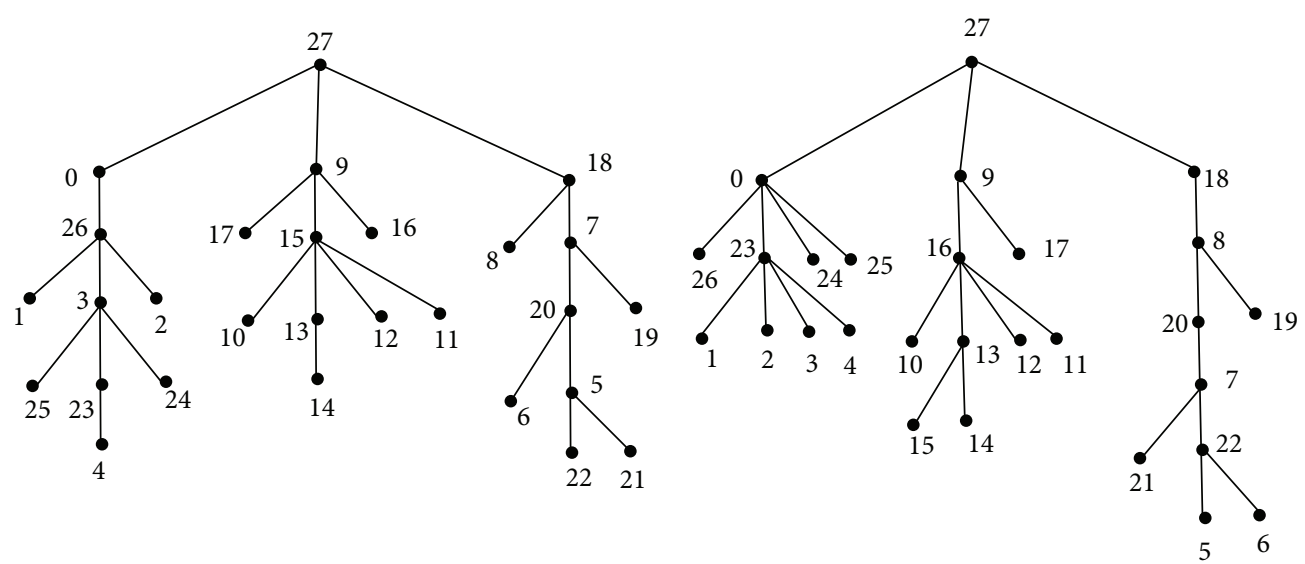

FIGURE 10: Six graceful caterpillars grouped into two, each containing three caterpillars.

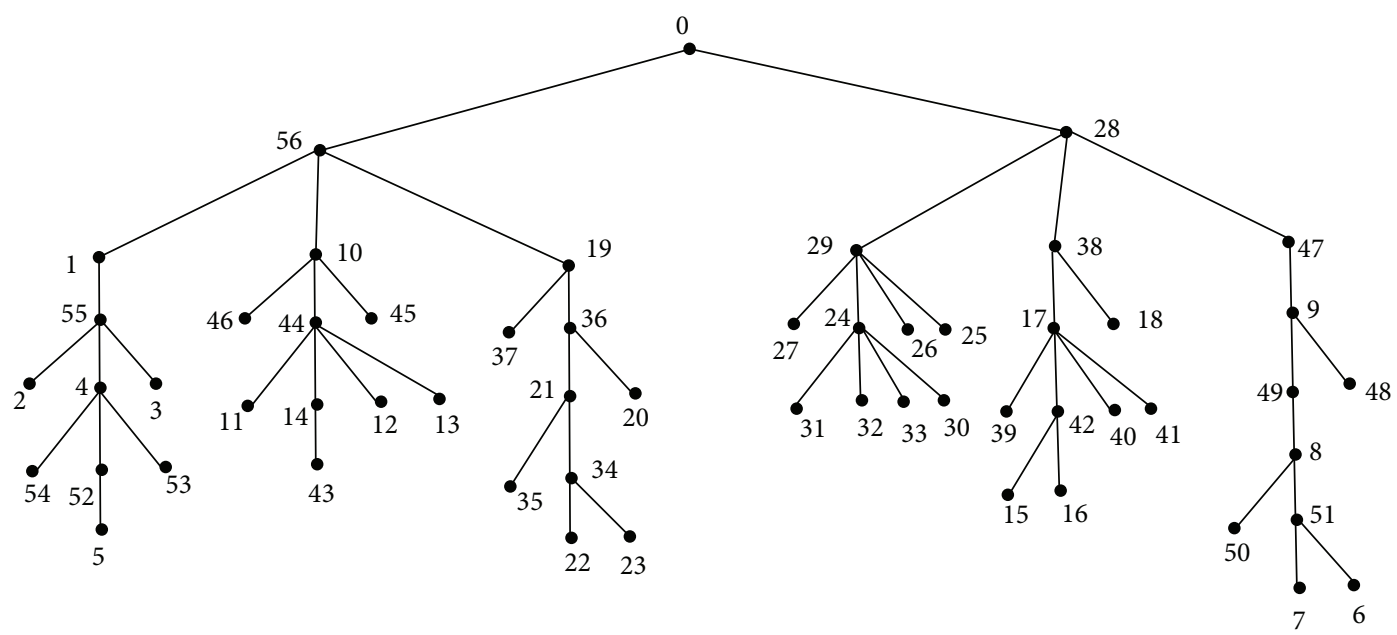

FIGURE 11: Both groups joined with a single root vertex. 
Theorem 4. All extended super-caterpillars are graceful.

Proof of Theorem 4. Theorem 2 asserts that we can label each group $i$ of caterpillars with root $v_{i}, \forall i=1, \ldots, k$, gracefully. Root $v$, connected to all $v_{i}$, is labeled 0 and labels of the remaining vertices in the tree are incremented by one. Let $s$ be the total number of vertices in each group (where $s=p m+1$ ). For group $i$, we will be labeling all odd level vertices by adding an offset $(k-i) s$ and for even level vertices $(i-1) s$ where $i=1,2,3, \ldots, k$. So $v_{i}$ gets label $(k-i) s$, and therefore edge $\left(v, v_{i}\right)$ gets label $(k-i) s$.

We will get the consecutive edge labels having the largest differences $(k-0) s-1$ to $(k-2) s+1$ in groups $T_{i}$ and $T_{(k-i+1)}$ for $i=1$ except $(k-1) s$ and $k s$ (Figure 9). Similarly, next consecutive differences $(k-2) s-1$ to $(k-4) s+1$ are found in $T_{i}$ and $T_{(k-i+1)}$ for $i=2$ again missing $(k-3) s$ and $(k-2) s$. So in general we can say that differences $(k-2(d-1)) s-1$ to $(k-2 d) s+1$ are generated while missing $(k-2 d+1) s$ and $(k-2(d-1)) s$ where $d=1,2, \ldots,\lceil k / 2\rceil$. These numbers are multiples of $s$ which have already been generated in edges $\left(v, v_{i}\right), i=1, \ldots, k$.

The following examples show how this labeling is done in an extended super-caterpillar consisting of six caterpillars as in Figure 7 grouped in three (Figure 8) or two (Figure 10) $(k=2$ or $k=3)$. Here each caterpillar has 9 vertices $(m=9)$. Then both of these groups are connected with $v$ and gracefully labeled as in Figures 9 and 11.

\section{Conclusion}

We have proved that two fairly general classes of trees are graceful. In particular, if a number of caterpillars have equal number of vertices and total number of vertices in odd levels of each caterpillar are equal, then such caterpillars can be joined to a vertex to obtain a graceful tree. In our future work, we would like to see if more generalized trees constructed from caterpillar can be gracefully labeled.

\section{Conflict of Interests}

The authors declare that there is no conflict of interests regarding the publication of this paper.

\section{References}

[1] C. Huang, A. Kotzig, and A. Rosa, "Further results on tree labellings," Utilitas Mathematica, vol. 21, pp. 31-48, 1982.

[2] A. Rosa, On Certain Valuations of the Vertices of a Graph, Theory of Graphs, International Symposium, Rome, Italy, 1967.

[3] E. Robeva, An extensive survey of graceful trees [Undergraduate Honours thesis], Stanford University, 2011.

[4] J. A. Gallian, "A dynamic survey of graph labeling," Dynamic Survey, vol. 6, article 43, 1997.

[5] K. Eshghi and P. Azimi, "Applications of mathematical programming in graceful labeling of graphs," Journal of Applied Mathematics, vol. 2004, no. 1, pp. 1-8, 2004.

[6] R. E. Aldred and B. D. McKay, "Graceful and harmonious labellings of trees," Bulletin of the Institute of Combinatorics and its Applications, vol. 23, pp. 69-72, 1998.
[7] T. Grace, "On sequential labelings of graphs," Journal of Graph Theory, vol. 7, no. 2, pp. 195-201, 1983.

[8] J. A. Gallian, "Living with the labeling disease for 25 years," Journal of the Indonesian Mathematical Society, pp. 45-58, 2012.

[9] W. Fang, "A computational approach to the graceful tree conjecture," CoRR Abs abs/1003.3045, 2010.

[10] G. S. Bloom and S. W. Golomb, "Applications of numbered undirected graphs," Proceedings of the IEEE, vol. 65, no. 4, pp. 562-570, 1977.

[11] G. S. Bloom and S. W. Golomb, Numbered Complete Graphs, Unusual Rulers and Assorted Applications, vol. 642, Springer, 1978.

[12] A. Rosa, "On certain valuations of the vertices of a graph," in Theory of Graphs, International Symposium, Rome, July 1966, pp. 349-355, Gordon and Breach, New York, NY, USA, 1967.

[13] J.-C. Bermond and J. Schönheim, "G-decomposition of $K_{n}$, where $G$ has four vertices or less," Discrete Mathematics, vol. 19, no. 2, pp. 113-120, 1977. 


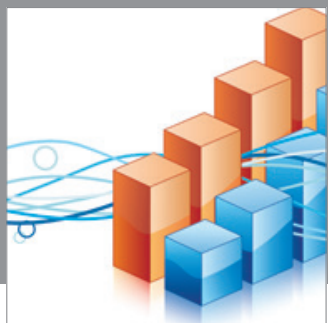

Advances in

Operations Research

mansans

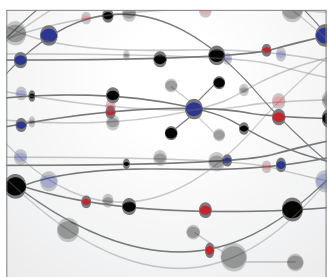

The Scientific World Journal
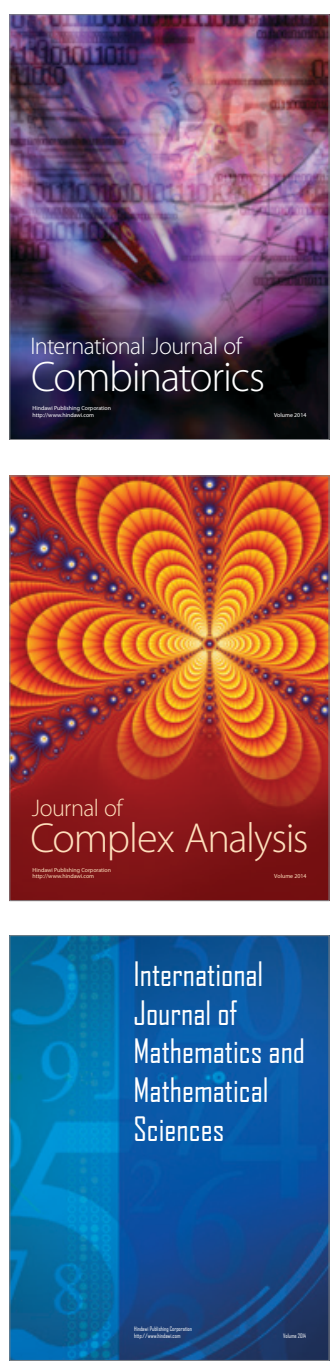
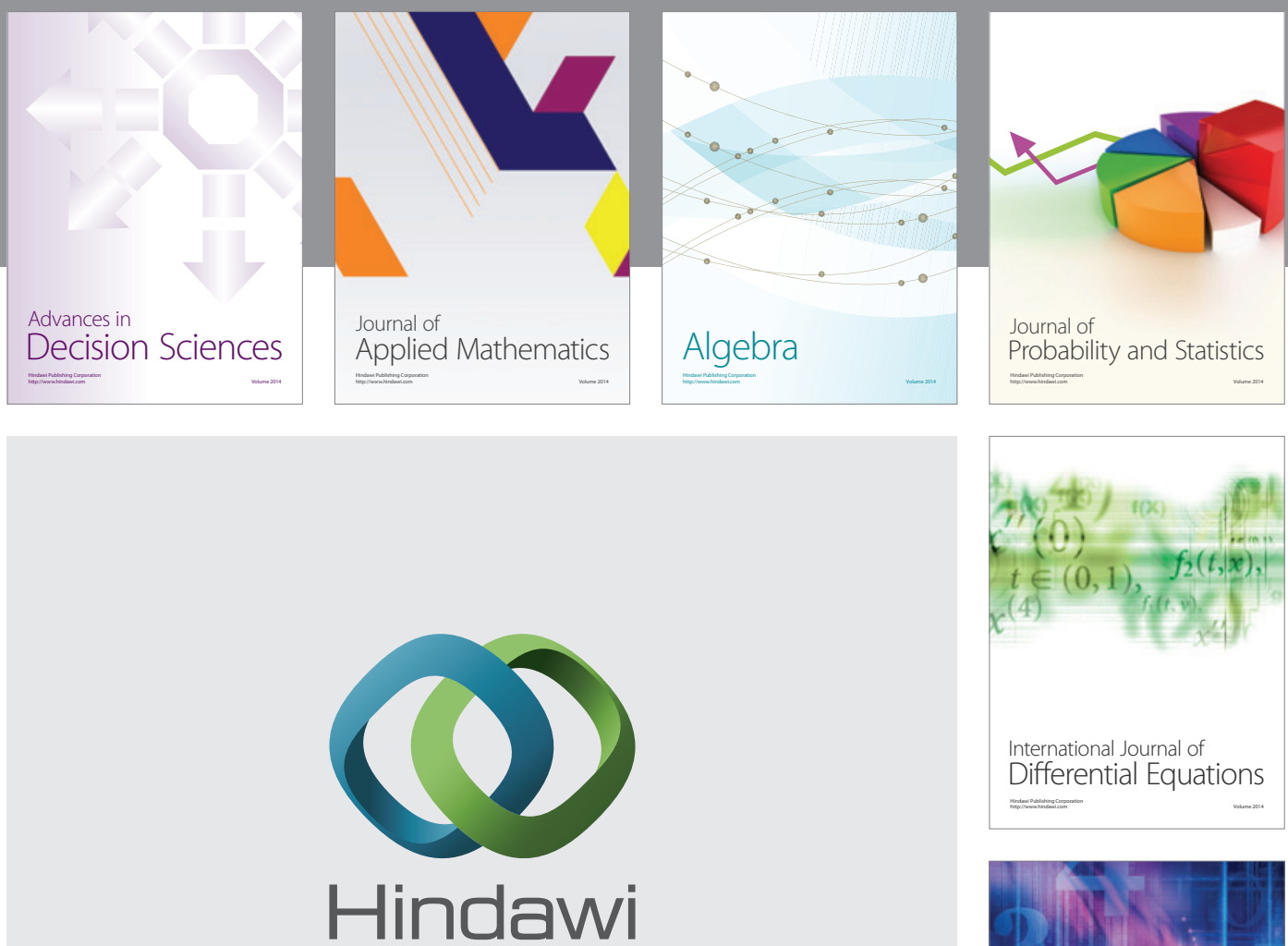

Submit your manuscripts at http://www.hindawi.com
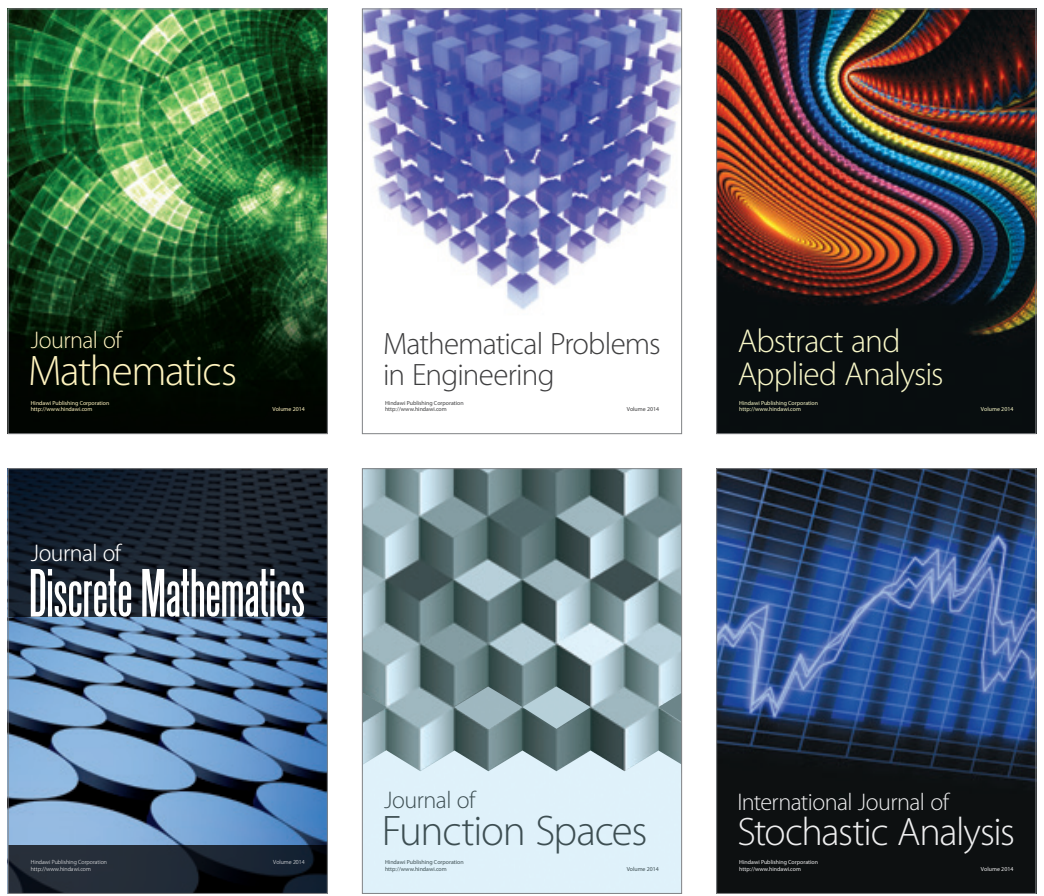

Journal of

Function Spaces

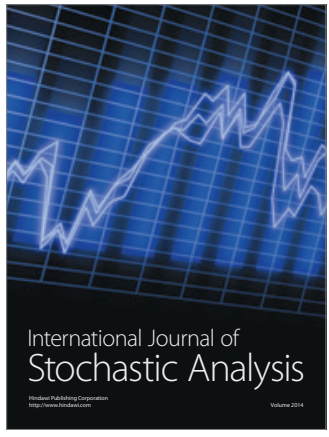

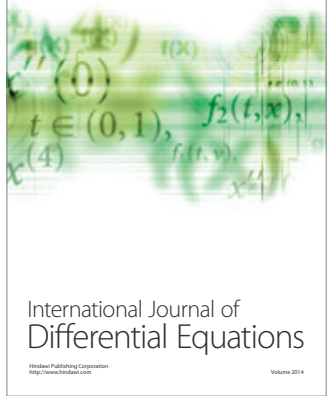
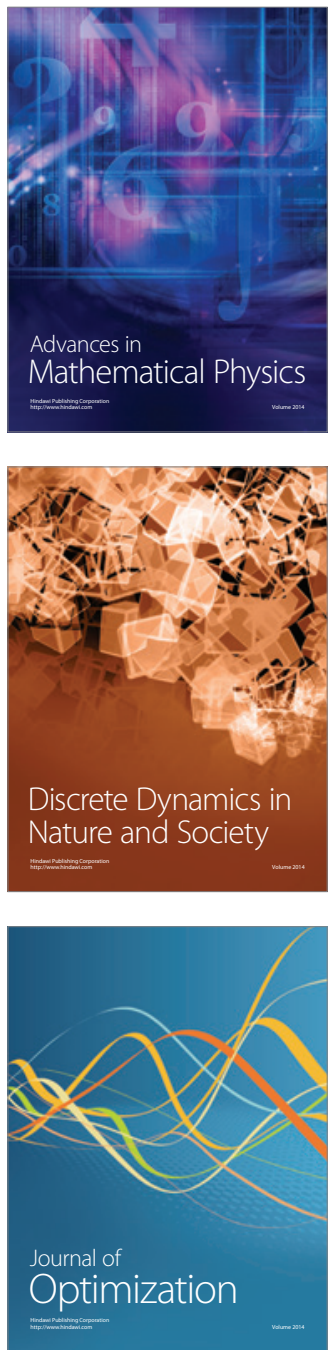